\title{
Use of a Hepatitis C Screening Order Set to Improve Screening Rates and Measure Intervention Effectiveness
}

Victoria L. Prince, MD, PhD | Sean McNamara, MD | Ken Gondor | Susan Pohl, MD | Osman Sanyer, MD | Kara A. Frame, MD

PRiMER. 2019;3:13.

Published: 4/12/2019 | DOI: 10.22454/PRiMER.2019.282710

\section{Abstract}

Introduction: Hepatitis C virus (HCV) infection leads to significant morbidity and mortality. Rates of HCV infection are greatest in patients born from 1945 to 1965, so the Centers for Disease Control recommends a one-time screening in this cohort. Previous interventions utilizing the electronic medical record (EMR) capabilities at two University of Utah Family Medicine clinics have increased screening rates significantly, but further improvement is possible.

Methods: A family medicine resident-led continuous quality improvement (CQI) team used the Model for Improvement methods popularized by the Institute for Healthcare Improvement to create a team-based intervention with the goal of improving HCV screening in a family medicine faculty and resident clinic. An order set was created and a protocol developed that allowed medical assistants or clinic phlebotomists to order the appropriate HCV screening lab if this had not yet been done by the primary care provider. Data were extracted from the EMR that showed changes in total and monthly screening rates as well as the frequency of order set use.

Results: Monthly screening rates at the Madsen Family Medicine Clinic (Salt Lake City, UT) increased from approximately $40 \%$ to greater than $50 \%$ in the 5 -month intervention period. The order set was used 19 times during this period which accounted for $18.8 \%$ of new screens.

Conclusions: Creating an order set that allows medical assistants to order the HCV screening lab increased HCV screening rates in our clinic. Because order set utilization data can be extracted from the EMR, this intervention provided a process measure that can differentiate the effect of this intervention from the effects of other interventions previously undertaken in the clinic.

\section{Introduction}

Rates of hepatitis $\mathrm{C}$ virus (HCV) infection are highest in patients born from 1945 to 1965 . In this cohort the Centers for Disease Control (CDC) recommends a one-time screening test for HCV. ${ }^{1}$ The incidence of HCV in Utah is the same as the national average, with an incidence of 51.1 per 100,000 in $2014 .{ }^{2}$ The CDC published this HCV screening recommendation in August 2012, ${ }^{3}$ yet as of January 2016 only 18\% of our clinic's established patients born from 1945-1965 ("the cohort") had been appropriately screened.

Previous studies have shown that utilizing electronic medical records (EMR) can improve HCV screening rates. ${ }^{4-8}$ Cohort screening rates were previously improved at two University of Utah Family Medicine faculty/resident clinics 
by adding HCV screening to the health maintenance reminders of the EMR and sending an electronic message through the patient portal to all cohort patients enrolled in the portal. After these interventions, monthly screening rates (the percentage of patients in the cohort seen each month who were appropriately screened) increased from $22.4 \%$ to $41 \%$. By October $2016,30.1 \%$ of all target patients in the cohort at these faculty/resident clinics had been screened.

Building on the efforts of these previous interventions, a resident-led continuous quality improvement (CQI) team was created to further improve screening rates. The addition of a new CQI project closely following the previous interventions created a challenge for data analysis, as the previous changes were likely still increasing screening rates. The creation of an HCV order set was a new intervention that built on the previous work. It allowed the CQI team to continue to improve HCV screening rates and its effectiveness could be measured by determining the frequency with which the medical assistant order set was used to complete HCV screening.

\section{Methods}

The CQI team was led by a third-year family medicine resident and consisted of residents, faculty, medical assistants (MAs), nurse practitioners, nurses, and a fellow. The team met monthly during structured CQI time at the University of Utah Family Medicine Clinic Quality Meetings. The CQI team followed the Model for Improvement ${ }^{9}$ methods to create the intervention. Work done by the CQI teams at the University of Utah Family Medicine Residency was reviewed (IRB_\#00050738) and determined not to meet the definitions of human subjects research.

There are two family medicine faculty and resident practices at the University of Utah. This CQI project was implemented at only one of those clinics, the Madsen Family Medicine Clinic. The other clinic underwent a different CQI intervention and is thus not appropriate as a control group for comparison.

The order set consists of an HCV antibody with reflex to HCV polymerase chain reaction confirmation blood test that could be ordered by an MA or phlebotomist if three screening questions were completed in the order set. The three questions were:

- Were you born between the years 1945-1965?

- Have you previously been screened for hepatitis C?

- Would you like to have an HCV screening drawn today?

If the responses to these questions resulted in the indication and inclination to proceed with testing, the medical assistant (MA) or phlebotomist could order the HCV screen. Physicians could also continue to order the lab through the normal order entry. The physician-placed order did not use the same MA order set. The order set was approved by the University of Utah Compliance office and the order set was created in Epic EMR system used by University of Utah Hospital and Clinics. The order set was introduced to clinic personnel at a monthly clinic quality meeting and medical assistants and providers who were part of the project team promoted the order set to their colleagues by word of mouth.

Data for this project were collected monthly by a database analyst at the University of Utah. The study included all patients born 1945-1965 with a primary care physician at the clinic who had been seen in the past 3 years. Monthly screening rates in this cohort were calculated by dividing the number of cohort seen each month who had received a one-time HCV screening by the total number of patients in the cohort seen that month. Information on when the HCV order set was used was also extracted. Data for this project was collected and analyzed for a 5-month time period from November 2016-March 2017. The primary outcome measure was the percentage of patients in the cohort seen who were appropriately screened for HCV. The secondary process measure was the number of HCV screens ordered by MAs or phlebotomists using the order set.

\section{Results}

As of March 1, 2017, 1,043 patients were in the cohort at the Madsen Clinic. Between November 2016 and March 2017, the total percentage of the cohort that had been screened for HCV at the Madsen Clinic increased from $30.1 \%$ 
to $40.5 \%$. During the study period, 144 to 181 members of the cohort were seen in clinic each month and the monthly screening rate increased from approximately $40 \%$ to greater than $50 \%$. Figure 1 shows the monthly screening rate had already improved with previous interventions. This outcome measure continued to rise with the addition of the MA order set.

Table 1 shows how many times the MA order set was used each month and what percentage of new screenings were completed as a result of the order set.

\section{Discussion}

During this 5-month, resident-led CQI project, monthly screening rates increased from approximately $40 \%$ to $50 \%$. During the study period the percentage of the cohort who had ever been screened increased from $30.1 \%$ to $40.5 \%$. Two previous CQI projects in our practice utilizing the EMR had already increased the HCV screening rates. Initial interventions included the addition of HCV screening to the EMR health maintenance tab and an electronic message through the EMR-linked patient portal to all patients due for screening. These interventions approximately doubled the monthly screening rate from around $20 \%$ to around $40 \%$. With this increase in monthly screenings, the total percentage of the cohort who had been screened increased from $18.3 \%$ to $30.1 \%$ between October 2015 and October 2016.

The development of an intervention that could be measured independently in the EMR allowed the authors to interpret the effect of the current CQI project separate from the effects of the previous interventions. By developing an order set that was designed to be used by MAs and phlebotomists, we increased screening in patients whose primary care providers had not ordered the screen.

The frequency with which the order set was used decreased over our study period. This may be due to a decline in the initial enthusiasm for the project among the MA and phlebotomists. Additionally, personnel changes in our clinic staff may have resulted in a decrease in overall staff familiarity with the tool. Promotion of this order set to new MAs and at the point of blood draw may increase its utilization.

Limitations of this study include its reliance on a specific EMR for implementation; however the concept is adaptable to other EMRs and could be implemented as a paper-based protocol. Additionally, this project focused on $\mathrm{HCV}$ screening, but similar protocols could be developed for other screening guidelines. Furthermore, this study was limited to a 5-month intervention period, and the long-term impact is not known. Interventions that focus on provider and patient education and not on the EMR have been successful in other resident clinics. ${ }^{10}$ Structured HCV education in our clinic, or other clinics wishing to implement similar interventions, would likely improve screening rates and use of the order set. Interventions that combine EMR utilization with provider education have been shown to be effective, such as the study by Al-hihi et al that showed an increase in age-recommended HCV screening from $30 \%$ to $55 \%$ in the University of Kansas Health System General Internal Medicine Department using EMR-based alerts and HCV-focused provider education as two separate interventions. ${ }^{11}$ Notably, in that study there was no process measure to determine which intervention was responsible for increases in screening rates once both interventions were active.

Our study showed increased screening rates and demonstrated the benefit of using a measurable intervention. Specifically, this order set allows data to be extracted from the EMR to measure the frequency with which the intervention was used and thus determine its role in increasing screening rates. As CQI interventions are often done in rapid succession, developing process or outcome measures that are unique to an intervention cycle is helpful in evaluating the efficacy of a new phase of a project.

\section{Tables and Figures}


Table 1:Medical Assistant Order Set Usage and Resultant Percentage of New Screenings

\begin{tabular}{|c|c|c|c|}
\hline Month & Order Set Use & New Screens & \% of Patients Screened by Order Set \\
\hline Nov-16 & 5 & 17 & $29.41 \%$ \\
\hline Dec-16 & 5 & 21 & $23.81 \%$ \\
\hline Jan-17 & 3 & 19 & $15.79 \%$ \\
\hline Feb-17 & 2 & 21 & $9.52 \%$ \\
\hline Mar-17 & 4 & 23 & $17.39 \%$ \\
\hline Total & 19 & 101 & $18.81 \%$ \\
\hline
\end{tabular}

Figure 1: Hepatitis C Monthly Screening Rates

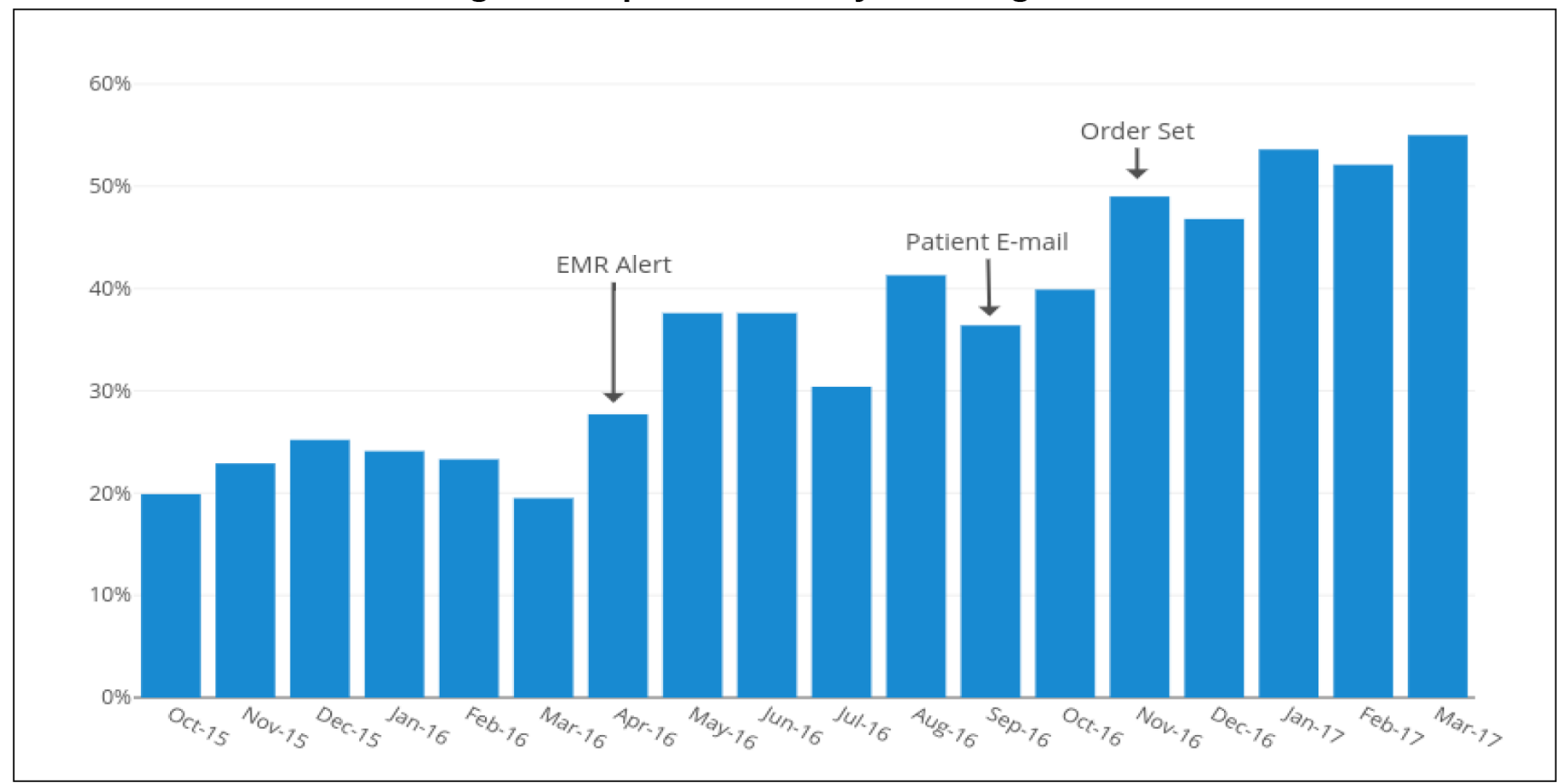

\section{Acknowledgments}

Presentations: This research was presented as a poster at the Society of Teachers of Family Medicine Annual Spring conference in San Diego, CA, May 5-9, 2017, and at the Ogden Medical-Surgical Conference in Ogden, UT, May 19, 2017.

\section{Corresponding Author}

Victoria L. Prince, MD, PhD

Department of Family and Preventive Medicine, 375 Chipeta Way, Suite A, Salt Lake City, UT 84108. 801-581-7234.

victoria.prince@hsc.utah.edu

\section{Author Affiliations}

Victoria L. Prince, MD, PhD - University of Utah Department of Family and Preventive Medicine, University of Utah Medical School, Salt Lake City, UT

Sean McNamara, MD - University of Utah Department of Family and Preventive Medicine, University of Utah Medical School, Salt Lake City, UT

Ken Gondor - University of Utah Department of Family and Preventive Medicine, University of Utah Medical School, 
Salt Lake City, UT

Susan Pohl, MD - University of Utah Department of Family and Preventive Medicine, University of Utah Medical School, Salt Lake City, UT

Osman Sanyer, MD - University of Utah Department of Family and Preventive Medicine, University of Utah Medical School, Salt Lake City, UT

Kara A. Frame, MD - University of Utah Department of Family and Preventive Medicine, University of Utah Medical School, Salt Lake City, UT

\section{References}

1. Centers for Disease Control. Testing Recommendations for Hepatitis C Virus Infection. https://www.cdc.gov /hepatitis/hcv/guidelinesc.htm. Accessed September 21, 2017.

2. Utah Department of Health. Utah Communicable Disease Annual Report 2015. http://health.utah.gov/epi/data /annualreport/2014_CD_Annual_Rpt.pdf. Accessed April 5, 2019.

3. Smith BD, Morgan RL, Beckett GA, et al; Centers for Disease Control and Prevention. Recommendations for the identification of chronic hepatitis $C$ virus infection among persons born during 1945-1965. MMWR Recomm Rep. 2012;61(RR-4):1-32.

4. Shahnazarian V, Karu E, Mehta P. Hepatitis C: improving the quality of screening in a community hospital by implementing an electronic medical record intervention. BMJ Open Qual. 2015 Jan 1;4(1):u208549.w3409. https://doi.org/10.1136\%2Fbmjquality.u208549.w3409

5. Wurcel AG, Chen DD, Chui KKH, Knox TA. Tweak your order set! Implementation of modified laboratory order set improves Hepatitis $C$ virus screening rates in people living with human immunodeficiency virus. Open Forum Infect Dis. 2017 May 16 [cited 2017 Sep 20];4(2). Available from: http://www.ncbi.nlm.nih.gov /pmc/articles/PMC5569929/. Accessed Sept 20, 2017.

6. Markus J, Sadiq O, Nerenz D, et al. Marked impact of revised hepatitis C screening guidelines with electronic medical record prompt on screening of the "baby boomer cohort" by primary care physicians. Gastroenterology. 2017;152(5):S1167-S1168. https://doi.org/10.1016/S0016-5085(17)33905-7

7. Taylor BS, Hanson JT, Veerapaneni P, Villarreal R, Fiebelkorn K, Turner BJ. Hospital-based hepatitis C screening of baby boomers in a majority hispanic South Texas cohort: successes and barriers to implementation. Public Health Rep. 2016 May;131(2_suppl):74-83.

8. Barocas JA, Wang J, White LF, et al. Hepatitis C Testing Increased Among Baby Boomers Following The 2012 Change To CDC Testing Recommendations. Health Aff (Millwood). 2017;36(12):2142-2150. https://doi.org /10.1377/hlthaff.2017.0684

9. Langley GL, Moen R, Nolan KM, Norman CL, Provost LP. The Improvement Guide: A Practical Approach to Enhancing Organizational Performance. 2nd ed. San Francisco: Jossey-Bass Publishers; 2009.

10. Aamar $A$, Madhani $K$, Singh $P, C$ ia $D$, Hepatitis $C$ screening: quality improvement in a resident continuity clinic. Gastroenterology. 2017;152(5):S1168. https://doi.org/10.1016/S0016-5085(17)33906-9

11. Al-Hihi E, Shankweiler C, Stricklen D, Gibson C, Dunn W. Electronic medical record alert improves HCV testing for baby boomers in primary care setting: adults born during 1945-1965. BMJ Open Qual. 2017;6(2):e000084. https://doi.org/10.1136/bmjoq-2017-000084

Copyright $@ 2019$ by the Society of Teachers of Family Medicine 